\title{
Effects of Exogenous Nitric Oxide on Wheat Exposed to Enhanced Ultraviolet-B Radiation
}

\author{
Liyan Yang ${ }^{1}$, Rong Han ${ }^{1}$, Yi Sun ${ }^{2,3^{*}}$ \\ ${ }^{1}$ Shanxi Normal University, Linfen, China; ${ }^{2}$ Biotechnology Research Centre, Shanxi Academy of Agricultural Sciences, Taiyuan, \\ China; ${ }^{3}$ Key Laboratory of Crop Gene Resources and Germplasm Enhancement on Loess Plateau, Ministry of Agriculture, Taiyuan, \\ China. \\ Email: *sunyi692003@163.com
}

Received April 20 ${ }^{\text {th }}, 2013$; revised May 20 ${ }^{\text {th }}, 2013$; accepted June $10^{\text {th }}, 2013$

Copyright (c) 2013 Liyan Yang et al. This is an open access article distributed under the Creative Commons Attribution License, which permits unrestricted use, distribution, and reproduction in any medium, provided the original work is properly cited.

\begin{abstract}
We explored the use of exogenous nitric oxide (NO) on alleviating effects of UV-B light on winter wheat development. Triticum aestivum L. cv. Linyou 7287 seeds were irradiated with UV-B $\left(10.08 \mathrm{~kJ} \cdot \mathrm{m}^{-2} \cdot \mathrm{d}^{-1}\right)$ (enhanced UV-B) and watered with either water or $100 \mu \mathrm{mol} \cdot \mathrm{L}^{-1} \mathrm{SNP}$ solution. Plants were also watered with the SNP alone. The results showed that enhanced UV-B produced negative effects on seedling development. Leaf length decreased and seedling biomass dropped significantly compared with the control. Photochemical efficiency (Fv/Fm) dropped, and chlorophyll and carotenoid content as well as the ATPase activity declined. Content of UV-absorbing compounds and activity of the POD increased compared to the control. Application of the SNP, a NO donor partially protected wheat seedlings exposed to elevated UV-B radiation in that their leaf lengths and biomass accumulation were enhanced compared to the UV-B treatment alone. SNP also improved the contents of chlorophyll, carotenoid and UV-absorbing compounds in leaves. ATPase activity was enhanced but no influence on POD activity. Furthermore, the application of SNP alone showed a favorable effect on seedling growth compared with the control.
\end{abstract}

Keywords: Nitric Oxide; Seedling Development; UV-B Radiation; Wheat

\section{Introduction}

Plants are sessile photoautotrophic organisms and thus must constantly adapt to surrounding environmental factors for optimal growth and development. Ultraviolet-B (UV-B) radiation (wavelengths from 280 to $320 \mathrm{~nm}$ ) is an intrinsic part of the sunlight. There is evidence of adverse effects of UV-B on plants including DNA damage and biomass reduction [1-3], and enhanced UV-B radiation has potentially harmful or even detrimental effects. Chloroplast is the photosynthesis organelle which is very sensitive to UV-B radiation, upon high dosage of UV-B radiation, yellow spots or streaks appeared on the treated leaf surfaces, which are attributed to decreased chlorophyll content and the possible injury on chloroplast [4]. Excessive radiation may lead to over-saturation of the photosynthetic light reactions, which eventually cause photo inhibitory damage to the photosynthetic apparatus [5]. Photosystem II (PSII) and ATP synthase are two kinds of proteins complex in thylakoid membrane, and

${ }^{*}$ Corresponding author. the later are abundantly located on plasma membrane, inner mitochondrial membrane and thylakoid membrane and play an important role in photosynthesis reaction. The target of UV-B radiation is the membrane [6] which can be damaged by increased reactive oxygen species (ROS) in the plant cell. UV-B exposure has shown to increase ROS [7]. Higher plants have evolved different mechanisms to resist the harm of ROS. These mechanisms are based on metabolic compounds and enzymes, including UV-absorbing substances and reactive oxygen species.

Nitric oxide (NO) acts as a signaling molecule and mediates multiple physiological processes in plants [8]. NO confers protection against the herbicide diquat, drought, and salt stress [9-11]. When Arabidopsis thaliana were exposed to UV-B irradiation, endogenous NO is generated, which indicated the involvement of NO in UV-B stress [7] (Mackerness et al., 2001); flavonoid biosynthetic pathway was systemically induced by UV-B in a NO dependent way [12]; the NO is able to protect cells from the deleterious effects of oxidative stress contribut- 
ing with the antioxidant response [13]. In the present study, we examined the effects of the exogenous nitric oxide on wheat seedling growth exposed to enhanced UV-B radiation. We also investigated some physiological characters among them those of pigments, intrinsic photochemical efficiency and ATPase activity, POD activity was analyzed as well.

\section{Materials and Methods}

\subsection{Plant Material and Treatments}

Winter wheat (Triticum aestivum L. cv. Linyou 7287) seeds were provided by the Wheat Research Institute, Shanxi Academy of Agricultural Sciences (SAAS), People's Republic of China. They were selected for uniform size and sterilized for 10 min with $0.1 \% \mathrm{HgCl}_{2}$ and washed for $50 \mathrm{~min}$ with running water. Ninety seeds were cultured on wet filter paper in each petri dish (diameter $18 \mathrm{~cm}$ ) and watered daily in a growth chamber at $25^{\circ} \mathrm{C}$ and $70 \%$ relative humidity. There were 4 treatments, with 3 replications. One day later, just as seeds were germinating, irradiation treatment was applied according to the light/dark period as given (Table 1). At the same time, the seedlings were watered with $100 \mu \mathrm{mol} \cdot \mathrm{L}^{-1}$ sodium nitroprusside (SNP, a NO donor) in S and SB groups every day, and the same amount of distilled water was applied in CK and B groups under same condition. SNP solution was prepared immediately prior to use.

The UV-B radiation intensity was $10.08 \mathrm{~kJ} \cdot \mathrm{m}^{-2} \cdot \mathrm{d}^{-1}$. The spectral irradiance from the lamps was determined with an Optronics spectroradiometer (Model 742 Optronics Laboratories, Orlando, FL, USA). The UV-B radiation was generated by a filtered lamp (30 W, $297 \mathrm{~nm}$, Qin brand, Baoji Lamp Factory, Baoji City, China.). The lamps were hung on the top of the petri dishes and the desired irradiation was obtained by adjusting the distance between the lamps and the petri dishes.

\subsection{Plant Height and Biomass Measurement}

Eight days after treatment, twenty seedlings per replication were randomly chosen from each treatment. A total

Table 1. Light/dark period of irritation treatments.

\begin{tabular}{cccc}
\hline \multirow{2}{*}{ Treatments } & \multicolumn{2}{c}{ Light $\left(\mathrm{hr} \cdot \mathrm{d}^{-1}\right)$} & \\
\cline { 2 - 3 } & White light & UV-B irritation & \\
\hline CK & 8 & - & 16 \\
B & 8 & 8 & $\left.\mathrm{~d}^{-1}\right)$ \\
S & 8 & - & 16 \\
SB & 8 & 8 & 16 \\
\end{tabular}

CK: distilled water without ultraviolet-B radiation; B: distilled water with ultraviolet-B radiation; S: SNP without ultraviolet-B radiation; SB: SNP with ultraviolet-B radiation. of 60 seedlings were measured and means of leaf length, fresh weight, and dry weight were recorded.

\subsection{Chlorophyll, Carotenoid and UV-Absorbing Compounds Content, POD Activity Measurement}

Eight days after treatment, $0.5 \mathrm{~g}$ of leaves was frozen in liquid nitrogen, grounded to a powder and extracted with $100 \%$ acetone. The pigment extracts were centrifuged for 3 - 5 min to make the extract transparent. The content of chlorophyll and carotenoid were immediately assayed spectrophotometrically according to Lee [14] and were expressed in $\mathrm{mg} \cdot \mathrm{g}^{-1}$ fresh weight. UV-absorbing compounds content was measured according to Smith et al [15], sections were immersed in $5 \mathrm{ml}$ methanol: conc. $\mathrm{HCl}$ : water solution, at the end of the extraction period, a absorbance of the solution at $280-320 \mathrm{~nm}$ was determined using a UV/Visible scanning spectrophotometer, and the area under the curve integrated to give total absorbance in the UV-B wavelength band. POD activity was determined by measuring the increase rate in absorbance at $470 \mathrm{~nm}$ of a mixture containing $1 \mathrm{ml}$ of $50 \mathrm{mM}$ sodium phosphate buffer (pH7.0), $0.95 \mathrm{ml}$ of $0.2 \%$ 2-methoxyphenol, $1 \mathrm{ml}$ of $0.2 \%$ hydrogen peroxide and 0.05 $\mathrm{ml}$ of enzyme extractor distilled water as negative control.

\subsection{Chlorophyll Fluorescence Measurement}

The chlorophyll fluorescence was determined by a portable pulse-modulated fluorometer (PAM-2000, Walz, Effeltrich, Germany) with a far-red source adapter. The maximum photochemical efficiency of PSII was determined from the ratio of variable $(\mathrm{Fv})$ to maximum $(\mathrm{Fm})$ fluorescence $\left(\mathrm{Fv} / \mathrm{Fm}=\left(\mathrm{Fm}-\mathrm{F}_{0}\right) / \mathrm{Fm}\right)$ in leaves that had been dark-adapted for $30 \mathrm{~min}$. The minimal fluorescence level $\left(\mathrm{F}_{0}\right)$ with all PSII reaction center open was determined by measuring modulated light of $735 \mathrm{~nm}$, which was sufficiently low not to induce any significant variable fluorescence. The maximal fluorescence level (Fm) with all PSII reaction center closed was determined by a saturating pulse of $8000 \mu \mathrm{mol} \cdot \mathrm{m}^{-2} \cdot \mathrm{d}^{-1}$ in the dark-adapted leaves.

\subsection{ATPase Activity Measurement}

Eight days after treatment, activity of ATPase located in thylakoid membrane of leaves as well as in plasma membrane of root cell was measured according to Chen [16].

\subsection{Statistical Analysis}

Statistical significance was estimated at $P<0.05$ according to Duncan's multiple range test. All data give mean \pm SD. 


\section{Results}

\subsection{The Effect of SNP on Chlorophyll, Carotenoid and UV-Absorbing Compounds Content in Seedlings under UV-B}

Significant declined chlorophyll (chlorophyll a and b) and carotenoid contents appeared in B group compared to those in the control $(P<0.05)$, but they increased at the presence of SNP under UV-B radiation compared to UV-B alone. Application of SNP resulted in higher content of chlorophyll and carotenoid than the control though there was no significant difference. Content of UV-absorbing compounds in B group was significant higher $(P$ $<0.05)$ than the control, but lower $(P>0.05)$ than that in SB group. Application of SNP alone induced higher generation of UV-absorbing compounds than the control ( $P$ $<0.05)$ but less than that in B group $(P>0.05)$ (Table 2).

\subsection{The Effects of the SNP on Chlorophyll Fluorescent in Seedlings Exposed to Enhanced UV-B}

Photosynthesis is very vulnerable to UV-B treatment. To study the effect of SNP on photosynthesis under the UV$B$ treatment, we measured the maximum efficiency of PSII photochemistry Fv/Fm (Figure 1). After 8 days of UV-B radiation, Fv/Fm significantly declined $(P<0.05)$ compared with that of the control. Application of SNP could reverse the inhibition on PSII photochemistry where $\mathrm{Fv} / \mathrm{Fm}$ was enhanced significantly compared with that in the B group $(P<0.05)$; the highest $\mathrm{Fv} / \mathrm{Fm}$ was observed in the $\mathrm{S}$ group.

\subsection{The Effect of SNP on ATPase Activity in Seedlings under Enhanced UV-B}

The highest ATPase activity was observed in the S group either for that in thylakoid membrane of leaves or plasma membrane of roots (Figure 2); the lowest ATPase activeity was showed in the B group which was significantly lower than that of the control $(P<0.05)$. ATPase located in thylakoid membrane was more sensitive to ultraviolet-B radiation in that its activity was $27.6 \%$ lower than control versus $11.94 \%$ decreased in plasma membrane of root cell. Application of SNP showed a favorable effect on ATPase activity in both thylakoid membrane and plasma membrane of root cell under UV-B exposure.

\subsection{The Effect of SNP on POD Activity}

The POD activity was the highest in the S group, and it was significantly higher $(P<0.05)$ than that in the rest groups. Application of SNP didn't induce a higher activity of POD compared to B group, but they were significantly higher than that of the control $(P<0.05)$ (Figure 3).

\subsection{Effects of SNP on Leaf Length and Biomass of Seedlings under UV-B}

Leaf length was severely inhibited under UV-B radiation compared with that of the control $(P<0.05)$; the inhibittion on leaf length was significantly alleviated at the presence of SNP under ultraviolet-B radiation compared to that under UV-B alone. SNP alone showed more favorable effect than the control. Same tendency was also observed for the plant biomass in that fresh and dry weights were significantly decreased under ultraviolet-B radiation compared with those of control $(P<0.05)$, and they increased at the presence of SNP (Table 3).

\section{Discussion}

Plants use inducible mechanisms to defend themselves from environmental concerns, including metabolic and morphological changes. When maize seedlings were exposed to enhanced ultraviolet-B, shoot height was dwarfed [17], and in the present study, leaf growth of wheat seedling was inhibited significantly. A decrease in leaf elongation might well serve to decrease UV-B exposure, but might influence photosynthesis and reduce photosynthetic accumulation. SNP application partially alleviated the adverse effects of UV-B on leaf growth. Moreover, SNP alone resulted in favorable impact on leaf growth compared to that of the control.

Table 2. Content of chlorophyll, carotenoid and UV-B absorbing compounds in seedlings exposed to UV-B.

\begin{tabular}{|c|c|c|c|c|}
\hline Treatment & $\begin{array}{l}\text { Chlorophyll a content } \\
\left(\mathrm{mg} \cdot \mathrm{g}^{-1} \mathrm{FW}\right)\end{array}$ & $\begin{array}{l}\text { Chlorophyll b content } \\
\left(\mathrm{mg} \cdot \mathrm{g}^{-1} \mathrm{FW}\right)\end{array}$ & $\begin{array}{l}\text { Carotenoid content } \\
\left(\mathrm{mg} \cdot \mathrm{g}^{-1} \mathrm{FW}\right)\end{array}$ & $\begin{array}{l}\text { Content of UV-absorbing } \\
\text { compounds (\%) }\end{array}$ \\
\hline CK & $1.87 \pm 0.20^{\mathrm{ab}}$ & $2.16 \pm 0.19^{a b}$ & $4.87 \pm 0.45^{\mathrm{ab}}$ & $0.32 \pm 0.02^{c}$ \\
\hline S & $2.01 \pm 0.23^{\mathrm{a}}$ & $2.36 \pm 0.20^{\mathrm{a}}$ & $4.98 \pm 0.50^{\mathrm{a}}$ & $0.51 \pm 0.04^{\mathrm{ab}}$ \\
\hline B & $1.20 \pm 0.10^{c}$ & $0.86 \pm 0.07^{\mathrm{c}}$ & $2.58 \pm 0.24^{\mathrm{c}}$ & $0.64 \pm 0.04^{\mathrm{a}}$ \\
\hline SB & $1.32 \pm 0.11^{\mathrm{c}}$ & $0.98 \pm 0.08^{c}$ & $2.77 \pm 0.26^{c}$ & $0.71 \pm 0.06^{\mathrm{a}}$ \\
\hline
\end{tabular}

CK: distilled water without ultraviolet-B radiation; B: distilled water with ultraviolet-B radiation; S: SNP without ultraviolet-B radiation; SB: SNP with ultraviolet-B radiation. Values are means $\pm \mathrm{SD}(\mathrm{n}=3)$, and values in the same column followed by different letters are significantly different at $P<0.05$. 


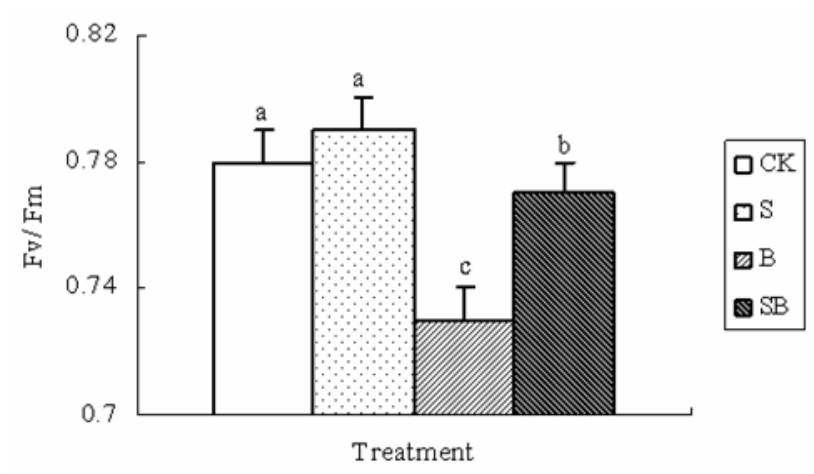

CK: distilled water without ultraviolet-B radiation; B: distilled water with ultraviolet-B radiation; S: SNP without ultraviolet-B radiation; SB: SNP with ultraviolet-B radiation. Each bar is the mean \pm SD $(n=3)$ for each treatment. Bars with different letter are significantly different at $P<0.05$.

Figure 1. The value of $\mathrm{Fv} / \mathrm{Fm}$ in various treatments.

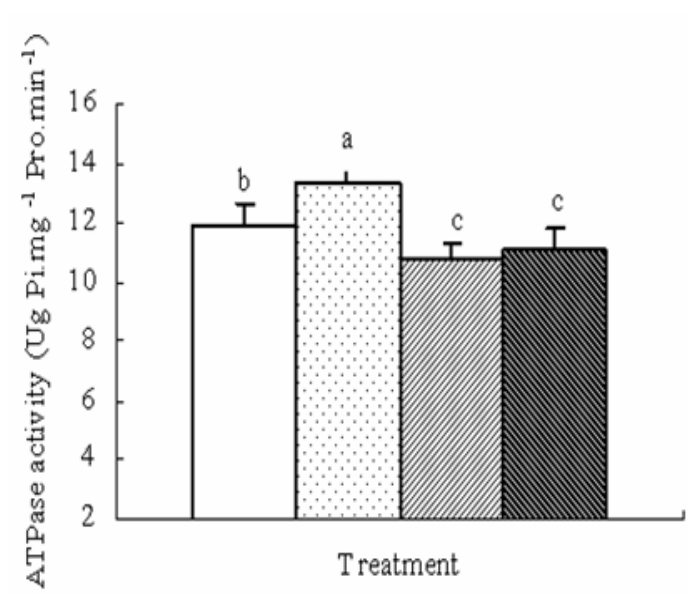

(a)
Antioxidative system is another inducible system upon UV-B radiation. Flavonoids and related phenolics are probably the most important UV-induced antioxidants which can absorb UV-B. In the present study, much more UV-absorbing substances were generated under UV-B radiation compared to control, SNP application induced more UV-B absorbing compounds, which means the more protection from UV-B, and SNP alone resulted in more antioxidant production. POD activity did not change with the SNP application compared to UV-B treatment alone. SNP alone induced improved POD activity which was consistent with the study of Costa and Shi $[18,19]$ and microarray studies where NO induces a large number of genes at transcriptional level; among them those of flavonoids related genes and antioxidant enzymes genes [20].

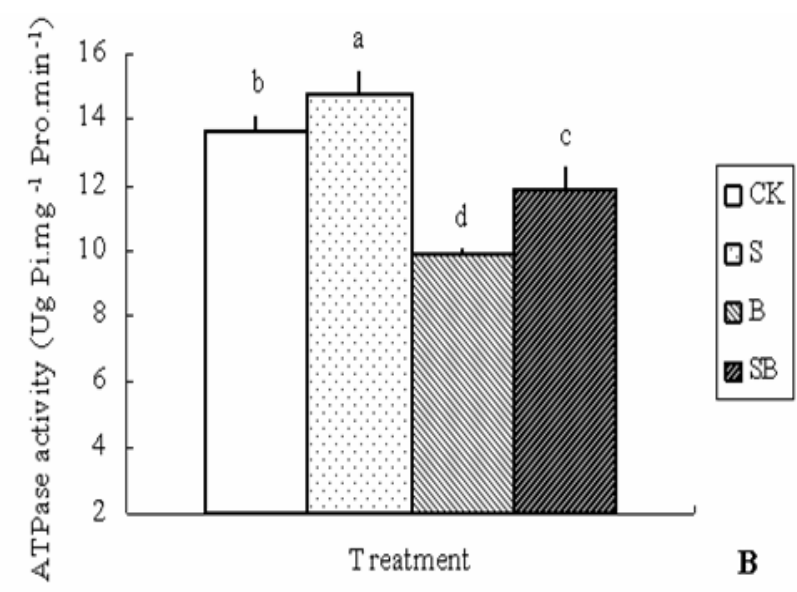

(b)

(a) Activity of ATPase that located in plasma membrane of seedling root; (b) Activity of ATPase that located in thylakoid membrane of wheat leaves. CK: distilled water without ultraviolet-B radiation; B: distilled water with ultraviolet-B radiation; S: SNP without ultraviolet-B radiation; SB: SNP with ultraviolet-B radiation. Each bar is the mean $\pm \mathrm{SD}(\mathrm{n}=3)$ for each treatment. Bars with different letter are significantly different at $P<0.05$

Figure 2. ATPase activity in various treatments.

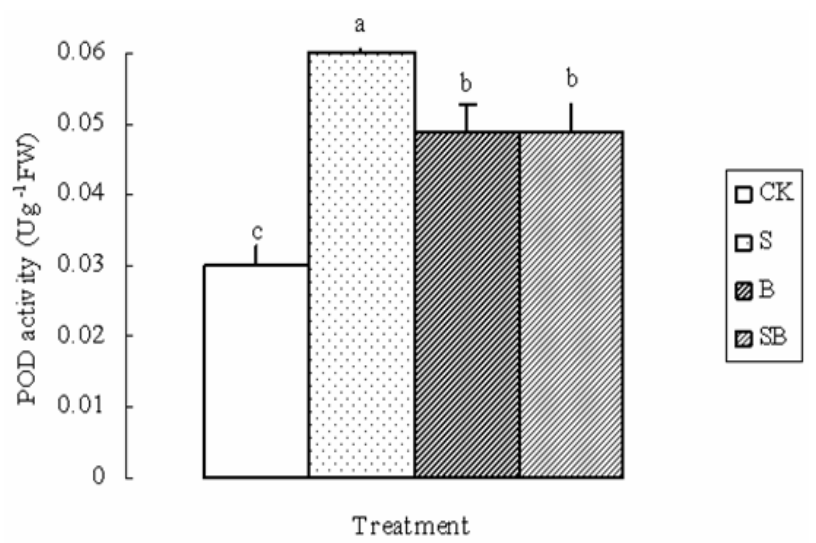

Each bar is the mean SD $(n=3)$ for each treatment. Bars with different letters are significantly different at $P<0.05$. CK: distilled water without ultraviolet-B radiation; $\mathrm{B}$ : distilled water with ultraviolet-B radiation; S: SNP without ultraviolet-B radiation; SB: SNP with ultraviolet-B radiation.

Figure 3. POD activity in various treatments.
Table 3. Leaf length and biomass in various treatments.

\begin{tabular}{cccc}
\hline Treatment & $\begin{array}{c}\text { Leaf length (cm) } \\
\text { (means } \pm \text { SD) }\end{array}$ & $\begin{array}{c}\text { Fresh weight (g) } \\
\text { (means } \pm \text { SD) }\end{array}$ & $\begin{array}{c}\text { Dry weight (g) } \\
\text { (means } \pm \text { SD) }\end{array}$ \\
\hline CK & $12.03 \pm 0.08^{\mathrm{a}}$ & $1.64 \pm 0^{\mathrm{a}}$ & $0.21 \pm 0.01^{\mathrm{a}}$ \\
S & $12.13 \pm 0.07^{\mathrm{a}}$ & $1.66 \pm 0.02^{\mathrm{a}}$ & $0.22 \pm 0.01^{\mathrm{a}}$ \\
B & $9.24 \pm 0.01^{\mathrm{c}}$ & $1.50 \pm 0.01^{\mathrm{b}}$ & $0.17 \pm 0.003^{\mathrm{b}}$ \\
& & & \\
SB & $10.35 \pm 0.02^{\mathrm{b}}$ & $1.56 \pm 0.04^{\mathrm{b}}$ & $0.19 \pm 0.007^{\mathrm{b}}$
\end{tabular}

CK: distilled water without ultraviolet-B radiation; B: distilled water with ultraviolet-B radiation; S: SNP without ultraviolet-B radiation; SB: SNP with ultraviolet-B radiation. Values are means \pm SD $(n=60)$, and values in the same column followed by different letters are significantly different at $P$ $<0.05$. 
Enhanced ultraviolet-B caused reduction of chlorophyll content and resulted in adaptive changes on photosynthetic apparatus such as thylakoid membrane, PSII system [4, 12]. In the present study, we found not only chlorophyll but also carotenoid content decreased under UV-B radiation. Carotenoid plays a vital role in the photosynthetic reaction centre where, it provides a mechanism for photo protection against auto-oxidation and they also participate in the energy-transfer process. The loss of carotenoid might negatively influence the photosynthesis. Measurement of the maximum efficiency of PSII photochemistry (Fv/Fm) revealed that it decreased under UV-B radiation. The ATP synthase of chloroplasts is an anabolic enzyme which is the prime producer of ATP, using the proton gradient generated by photosynthesis. ATP synthase activity of chloroplasts was significantly inhibited under UV-B radiation compared to that of the control, which implied that the ATP generation might be hampered accordingly. At the presence of SNP, the values of Fv/Fm were higher than that of UV-B treatment alone, so were the contents of chlorophyll and carotenoid, as well as ATPase activity. We attribute those favorable effects of SNP to following: NO, an important signaling molecule, involved in UV-B transduction pathway and plants may use exogenous NO as a protection strategy against elevated doses of UV-B. NO may fulfill this function by inducing more antioxidant to absorb UV-B. Decreased UV-B irradiation and peroxidant damage on photosynthetic apparatus as PSII and ATPase partially alleviated the inhibition on them caused by UV-B. We also conclude ATPases of root cell membrane was less sensitive to UV-B, but their activity was declined too, SNP alone showed a more favorable effects compared to the control.

\section{Acknowledgements}

This research project was supported by the Shanxi Scholarship Council of the People's Republic of China (2011061) and the National Nature Science Foundation of Shanxi Normal University (SMYKZ-18).

\section{REFERENCES}

[1] C. M. Rousseaux, S. D. Flint, P. S. Searles and M. M. Caldwell, "Plant Responses to Current Solar UltravioletB Radiation and Supplemented Solar Ultraviolet-B Radiation Simulating Ozone Depletion: An Experimental Comparison," Photochemistry and Photobiology, Vol. 80, No. 2, 2004, pp. 224-230.

[2] P. Majer and É. Hideg, "Developmental Stage Is an Important Factor That Determines the Antioxidant Responses of Young and Old Grapevine Leaves under UV Irradiation in a Greenhouse," Plant Physiology and Biochemistry, Vol. 50, 2011, pp. 15-23. doi:10.1016/j.plaphy.2011.09.018

[3] G. Fabón, L. Monforte, R. Tomás-Las-Heras, E. Núñez-
Olivera and J. Martínez-Abaigar, "Dynamic Response of UV-Absorbing Compounds, Quantum Yield and the Xanthophyll Cycle to Diel Changes in UV-B and Photosynthetic Radiations in an Aquatic Liverwort, " Journal of Plant Physiology, Vol. 169, No. 1, 2012, pp. 20-26. doi:10.1016/j.jplph.2011.08.010

[4] L. Y. Yang, R. Han and Y. Sun, "Damage Repair Effect of He-Ne Laser on Wheat Exposed to Enhanced Ultraviolet-B Radiation," Plant Physiology and Biochemistry, Vol. 57, 2012, pp. 218-221. doi:10.1016/j.plaphy.2012.06.003

[5] E. M. Aro, I. Virgin and B. Anersson, "Photoinhibition of Photosystem II: Inactivation, Protein Damage and Turnover," Biochimica et Biophysica Acta, Vol. 1143, No. 2, 1993, pp. 113-134. doi:10.1016/0005-2728(93)90134-2

[6] A. K. Jansen, V. Gaba and B. Greenberg, "Higher Plants and UV Radiation: Balancing Damage, Repair and Acclimation," Trends in Plant Science, Vol. 3, No. 4, 1998, pp. 131-135. doi:10.1016/S1360-1385(98)01215-1

[7] S. A. H. Mackerness, C. F. John, B. Jordan and B. Thomas, "Early Signaling Components in Ultraviolet-B Responses: Distinct Roles for Different Reactive Oxygen Species and Nitric Oxide,” FEBS Letters, Vol. 489, No. 2-3, 2001, pp. 237-242. doi:10.1016/S0014-5793(01)02103-2

[8] M. Leitner, E. Vandelle, F. Gaupels, D. Bellin and M. Delledonne, "NO Signals in the Haze: Nitric Oxide Signalling in Plant Defence," Current Opinion in Plant Biology, Vol. 12, No. 4, 2009, pp. 451-458. doi:10.1016/j.pbi.2009.05.012

[9] M. V. Beligni, and L. Lamattina, "Nitric Oxide Counteracts Cytotoxic Processes Mediated by Reactive Oxygen Species in Plant Tissues,” Planta, Vol. 208, No. 3, 1999, pp. 337-344. doi:10.1007/s004250050567

[10] C. Garcia-Mata and L. Lamattina, "Nitric Oxide Induces Stomatal Closure and Enhances the Adaptive Plant Responses against Drought Stress,” Plant Physiology, Vol. 126, No. 3, 2001, pp. 1196-1204. doi:10.1104/pp.126.3.1196

[11] C. L. Zeng, L. Liu, B. R. Wang, X. M. Wu and Y. Zhou, "Physiological Effects of Exogenous Nitric Oxide on Brassica juncea Seedlings under $\mathrm{NaCl}$ Stress,” Biologia Plantarum, Vol. 55, No. 2, 2011, pp. 345-348. doi:10.1007/s10535-011-0051-5

[12] V. Tossi, C. Lombardo, R. Cassia and L. Lamattina, "Nitric Oxide and Flavonoids Are Systemically Induced by UV-B in Maize Leaves,” Plant Science, Vol. 193-194, 2012, pp.103-109. doi:10.1016/j.plantsci.2012.05.012

[13] A. D. Boveris, A. Galatro and S. Puntarulo, "Effect of Nitric Oxide and Plant Antioxidants on Microsomal Content of Lipid Radicals,” Biological Research, Vol. 33, No. 2, 2000, pp. 159-165. doi:10.4067/S0716-97602000000200016

[14] H. S. Lee, "Principles and Experimental Techniques of Plant Physiology and Biochemistry,” Higher Education Press, Beijing, 2000. (in Chinese)

[15] J. L. Smith, D. J. Burritt and P. Bannister, "Shoot Dry Weight, Chlorophyll and UV-B-Absorbing Compounds as Indicators of a Plant's Sensitivity to UV-B Radiation,” Annals of Botany, Vol. 86, No. 6, 2000, pp. 1057-1063. 
doi:10.1006/anbo.2000.1270

[16] H. D. Chen, H. Xu and Y. W. Lian, "Experimental Techniques of Modern Plant Physiology," Science Press, Beijing, 2005. (in Chinese)

[17] M. Zhang, L. An, H. Feng, T. Chen, K. Chen, Y. H. Liu, H. G. Tang, J. F. Chang and X. L. Wang, "The Cascade Mechanisms of Nitric Oxide as a Second Messenger of Ultraviolet B in Inhibiting Mesocotyl Elongations,” Photochemistry and Photobiology, Vol. 77, No. 2, 2003, pp. 219-225.

doi:10.1562/0031-8655(2003)077<0219:TCMONO>2.0. $\mathrm{CO} ; 2$

[18] H. Costa, S. M. Gallego and M. L .Tomaro, "Effect of
UV-B Radiation on Antioxidant Defense System in Sunflower Cotyledons,” Plant Science, Vol. 162, No. 6, 2001, pp. 939-945. doi:10.1016/S0168-9452(02)00051-1

[19] S. Shi, G. Wang, Y. Wang, L. Zhang and L. Zhang, "Protective Effect of Nitric Oxide against Oxidative Stress under Ultraviolet-B Radiation,” Nitric Oxide, Vol. 13, No. 1, 2005, pp. 1-9. doi:10.1016/j.niox.2005.04.006

[20] M. Parani, S. Rudrabhatla, R. Myers, H. Weirich, B. Smith, D. W. Leaman and S. Goldman, "Microarray Analysis of Nitric Oxide Responsive Transcripts in Arabidopsis,” Plant Biotechnology Journal, Vol. 2, No. 4, 2004, pp. 359-366. doi:10.1111/j.1467-7652.2004.00085.X

\section{Abbreviation}

NO: nitric oxide; UV-B: Ultraviolet B; POD: peroxidase; SNP: sodium nitroprusside; ROS: reactive oxygen species; PSII: Photosystem II; ATPase: ATP synthase. 